\title{
Assessment of the use of earplugs effectiveness on a slow eating rate protocol
}

\author{
Filip Koidis ${ }^{1}$, Fyin Makinwa ${ }^{1}$, Kathryn Hart ${ }^{1}$, Michelle Gibbs ${ }^{1}$ and Shelagh Hampton ${ }^{2}$ \\ ${ }^{1}$ Department of Nutrition \& Metabolism and ${ }^{2}$ Department of Physiology and Biochemistry School of Biosciences and \\ Medicine, Faculty of Health and Medical Sciences, University of Surrey, Guildford, GU2 $7 X H$
}

Eating rate has gained the attention of scientific research due to its potential association with obesity and its co-morbidities ${ }^{(1)}$. Evidence to date supports the notion that eating rate affects energy intake. Reduction in eating rate may therefore offer a potential approach to reducing energy intake though evidence of its effects on satiety is limited. Effective and easy-to adopt interventions to reduce eating rate in everyday life are needed ${ }^{(2)}$. The aim of this study was to assess if the use of earplugs (as a means of reducing distraction) has an added benefit on slowing eating rate and improves concordance with protocol and perceived satiety.

Twelve healthy participants: (6 females, 6 males, age $24 \cdot 5$ [3.2], BMI $22 \cdot 1[3 \cdot 7] \mathrm{kg} / \mathrm{m}^{2}$ [SD], ) were randomised to a one-way crossover eating rate study. Following anthropometric measurements an isocaloric meal (606kcal, $37 \mathrm{~g}$ protein, $85 \mathrm{~g}$ carbohydrates, $12 \mathrm{~g}$ fat) was consumed during which the participants' normal eating rate (NER) was recorded. Participants received instructions on a slow eating rate protocol (SER), which was carried out on two separate occasions with (EARSIN) and without earplugs (EARSOUT). Each visit was separated by at least seven days. Eating rate (mouthfuls, chews per minute and swallows) was assessed independently by two researchers from front and side view video recordings. Perceived satiety was measured at regular intervals using electronic visual analogue scales (VAS) before and after consuming the set meal.

The data was tested for normality, repeated measures ANOVA with Tukey's post hoc test was completed .

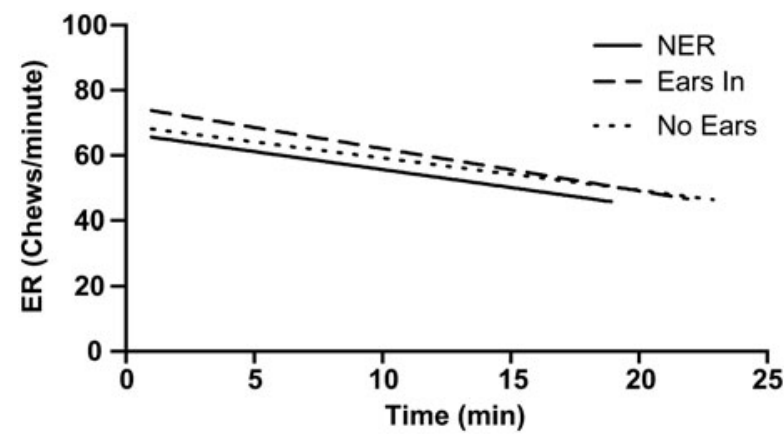

Eating Rate (chews/min) vs Time(min)

All participants showed a decelerated eating rate during NER which did not change significantly for the first seven comparable minutes $(p=0.389)$ when following the SER protocol with and without earplugs. For most participants following the SER protocol, their meal duration was doubled compared to the normal eating rate (NER 8.8 min, EARSIN 16.3 min, $p=0.001)$ with no significant differences between the use or absence of earplugs (EARSOUT $16.9 \mathrm{~min}$, EARSIN 16.3 min).

We have previously reported satiation results from this study demonstrating that the slow eating rate protocol significantly improved perceived satiety and that the use of earplugs had a beneficial addition ${ }^{(3)}$. However no significant differences in eating rate when following the SER protocol with or without headphones, despite the profound meal prolongation effect. The eating rate protocol may, in part, be affecting satiety through a biochemical pathway, and further eating rate studies are needed to investigate this association.

1. Bisogni CA, Falk LW, Madore E, et al. Dimensions of everyday eating and drinking episodes. Appetite. 2007; 48: 218-31

2. Robinson E, Almiron-Rig E, Rutters F, de Graaf C, FORDE CG, Tudue Smith C, Nolan SJ, Jebb SA. A systematic review and meta-analysis examining the effect of eating rate on energy intake and hunger. Am J Clin Nutr. 2014 Jul;100(1):123-51. doi: 10.3945/ajcn.113.081745.

3. Koidis F, Makinwa F, Gibbs M and Hampton F. Is satiation altered by earlplugs in an eating rate study. Abstract presented in: Satiety-from Origins to Applications, ASO Conference, 3rd March 2015. Appe 